explain this is being explored and numbers increased. The results may help determine the best methodology for assessing isolate susceptibility to a phage mix for inclusion in a future clinical trial.

\section{S113 AN EPIDEMIOLOGICAL REVIEW OF STRAINS OF PSEUDOMONAS AERUGINOSA IN A NON-CYSTIC FIBROSIS BRONCHIECTASIS COHORT}

${ }^{1} \mathrm{P}$ Mitchelmore, ${ }^{2} \mathrm{~A}$ Brown, ${ }^{3} \mathrm{C}$ Sheldon, ${ }^{1} \mathrm{C}$ Scotton, ${ }^{4} \mathrm{M}$ Bull, ${ }^{4} \mathrm{E}$ Mahenthiralingam, ${ }^{3} \mathrm{~N}$ Withers. ${ }^{1}$ University of Exeter Medical School, Exeter, UK; ${ }^{2}$ University of Exeter, Exeter, UK; ${ }^{3}$ Royal Devon \& Exeter Hospital, Exeter, UK; ${ }^{4}$ Cardiff University, Cardiff, UK

\subsection{6/thoraxjnl-2015-207770.119}

Introduction and objectives Pseudomonas aeruginosa $(\mathrm{Pa})$ is a significant respiratory pathogen. Research in Cystic Fibrosis cohorts has revealed transmissible strains, leading to heightened infection control protocols due to concerns of cross-infection. In patients with Non-Cystic Fibrosis Bronchiectasis (NCFB), the research is more limited. Our objectives were to investigate the strains found in our local NCFB population, and assess the occurrence of shared strains.

Methods Patients with NCFB and previous $\mathrm{Pa}$ in sputum culture consented to providing sputum for the study and review of their medical notes. Sputum samples from patients were processed in the usual manner and if $\mathrm{Pa}$ was isolated, 10 representative colonies per patient were stored for strain typing. Isolates were subjected to Random Amplification of Polymorphic DNA (RAPD). Distinct RAPD types were verified by electrophoresis on an Agilent Bioanalyzer and subsequent cluster analysis using GelCompar II software, and further investigated by Multi-Locus Sequence Typing (MLST).

Results $\mathrm{Pa}$ was obtained from 46 patients over 12 months providing 459 isolates. Co-existence of multiple strains was observed in two patients. Twenty patients (43\%) had unique strains by RAPD and the remaining patients were clustered into 7 subgroups, defined as $\geq 90 \%$ homology by RAPD, using Pearson's correlation analysis. The largest cluster showed a predominance of one MLST strain type identified as ST-17 (also known as "Clone C") on the MLST database. In our cohort, 8 patients $(17 \%)$ harboured Clone C, which is a higher prevalence than observed in previous UK studies of various patient cohorts (typically 2-6\% prevalence). MLST analysis of smaller RAPD clusters identified other MLST strain types shared by 2 or 3 patients. As with Clone C, all the observed shared MLST strain types are globally distributed. MLST did not reveal any novel shared strains.

Conclusions Our cohort of patients with NCFB shows evidence of shared strains of $\mathrm{Pa}$ including a high prevalence of Clone $\mathrm{C}$ compared to previous national reports. Whilst the occurrence of shared strains may reflect their global distribution, we cannot rule out cross-infection between patients.

\section{S114 FEASIBILITY STUDY FOR A RANDOMISED CONTROLLED TRIAL OF PSEUDOMONAS AERUGINOSA ERADICATION TREATMENT IN PATIENTS WITH BRONCHIECTASIS}

${ }^{1} \mathrm{HR}$ Abo-Leyah, ${ }^{2} \mathrm{~A}$ Smith, ${ }^{3} \mathrm{M}$ Clark, ${ }^{2} \mathrm{~J}$ Hill, ${ }^{1} \mathrm{TC}$ Fardon, ${ }^{1} \mathrm{JD}$ Chalmers. ${ }^{1}$ Department of Respiratory Medicine, College of Medicine, University of Dundee, Dundee, UK; ${ }^{2}$ College of Medicine, University of Dundee, Dundee, UK; ${ }^{3}$ Department of Microbiology, College of Medicine, University of Dundee, Dundee, UK

10.1136/thoraxjnl-2015-207770.120
Introduction Guidelines recommend Pseudomonas aeruginosa (PA) eradication treatment for new isolation of PA in bronchiectasis patients, but evidence is lacking. We performed a feasibility study to identify how many patients with new PA isolation would be eligible for a future randomised controlled trial (RCT). Methods For 12 months (2013-2014) we piloted a PA "alert" system that would notify the study team when PA was isolated in sputum samples. Patients were reviewed using electronic medical records to classify patients as 1- New PA, having never isolated PA before and therefore eligible for eradication, 2- Relapsed PA, patients having been free from PA and now isolating it againtherefore eligible for eradication, 3- Chronic PA, and therefore not eligible for eradication, 4- patients without HRCT confirmed bronchiectasis. Anti-PA IgG antibodies were measured in serum as a predictor of potential eradication success.

Results There were $322 \mathrm{PA}$ isolates from sputum over 12 months, in 156 patients. 22 patients presented new PA, 13 patients relapsed PA, 17 chronic PA and 104 patients did not have bronchiectasis (66\%). The most frequent diagnoses in the non-bronchiectasis group was COPD.

Overall, 35 patients would have been eligible for a trial of PA eradication on clinical grounds. Of these patients, clearance (negative PA culture at next follow-up sample) was demonstrated in $63 \%$.

PA IgG was positive in all of the patients with chronic PA, and identified those with successful clearance: sensitivity $93 \%$ and specificity $54 \%$. A low antibody level was strongly associated with successful eradication (negative likelihood ratio 0.06, successful eradication in 92.3\%).

Mortality was very high in this patient group. 9\%, 30\% and $24 \%$ of patients died within 12 months follow-up in the 3 groups respectively.

Conclusion This feasibility demonstrates that a future community based RCT of PA eradication would require a large number of centres, and be resource intensive. Major challenges in powering a future study include the low proportion of true first isolates and the high frequency of spontaneous PA clearance. Raised PA IgG was not sufficiently specific to exclude successful eradication, but a low PA IgG suggests a high likelihood of success.

\section{S115 EFFICACY OF PSEUDOMONAS AERUGINOSA ERADICATION REGIMENS IN NON-CF BRONCHIECTASIS}

${ }^{1} \mathrm{E}$ Vallieres, ${ }^{2} \mathrm{~K}$ Tumelty, ${ }^{3} \mathrm{MM}$ Tunney, ${ }^{2} \mathrm{R}$ Hannah, ${ }^{2} \mathrm{O}$ Hewitt, ${ }^{1} \mathrm{JS}$ Elborn, ${ }^{2} \mathrm{DG}$ Downey. ${ }^{1}$ Queen's University Belfast, School of Medicine, Dentistry and Biomedical Sciences, Belfast, UK; ${ }^{2}$ Belfast City Hospital, Belfast Health and Social Care Trust, Belfast, UK; ${ }^{3}$ Queen's University Belfast, School of Pharmacy, Belfast, UK

\subsection{6/thoraxjnl-2015-207770.121}

Introduction and objectives BTS guidelines recommend to try and eradicate Pseudomonas aeruginosa when first isolated from patients with non-CF bronchiectasis. The aims of this study were to 1 ) define the different eradication regimens used in our respiratory centre and 2) evaluate their efficacy.

Methods The medical notes of adult non-CF bronchiectasis patients who underwent eradication trial for $P$. aeruginosa, between 2007 and 2014, were retrospectively reviewed. Eradication was considered successful if all (and at least 3 ) respiratory samples collected during the 6-month period following initiation of eradication were free of $P$. aeruginosa.

Results During the study period, 67 patients $(58 \%$ male, average age $63.0 \mathrm{yrs}$ ) had at least one eradication trial. The majority of 